International Journal of Food Science, Nutrition and Dietetics (IJFS)

ISSN 2326-3350

\title{
Ingestion of Tabebuia avellanedae (Taheebo) Inhibits Production of Reactive Oxygen Species from Human Peripheral Blood Neutrophils
}

Research Article

Ohno $\mathrm{S}^{1,2}$, Ohno $\mathrm{Y}^{1}$, Suzuki $\mathrm{Y}^{3}$, Miura $\mathrm{S}^{4}$, Yoshioka $\mathrm{H}^{4}$, Mori $\mathrm{Y}^{4}$, Suzuki $\mathrm{K}^{1,5^{*}}$

${ }^{1}$ Institute for Nanoscience \& Nanotechnology, Waseda University, Tokyo, Japan.

${ }^{2}$ Department of Integrative Medicine, Graduate School of Medicine, Osaka University, Osaka, Japan.

${ }^{3}$ Collaborative Major in Advanced Health Science, Tokyo University of Agriculture and Technology / Waseda University, Tokyo, Japan.

${ }^{4}$ Mebiol Inc., Kanagawa, Japan.

${ }^{5}$ Faculty of Sport Sciences, Waseda University, Saitama, Japan.

\section{Abstract}

Neutrophils are a main source of oxidative stress due to reactive oxygen species (ROS) production, and the tissue damage caused by excessive ROS might lead to aging and a variety of diseases. Whereas the anti-inflammatory effects of Tabebuia avellanedae (taheebo) have been reported in vitro and in animal experiments, little is known about the effects of taheebo in humans. Fourteen adult volunteers ingested $500 \mathrm{mg}$ of taheebo extract in 4 capsules (Taheebo Japan, Co., Ltd., Osaka, Japan) daily for 2 weeks. The production of ROS from peripheral blood neutrophils was measured by luminol-dependent chemiluminescence $(\mathrm{LmCL})$ in a kinetic mode at 30-minute intervals for 1.5 hours with a luminometer at $37^{\circ} \mathrm{C}$. We found that, peak and sum of values of LmCL were significantly decreased at the 1.5 hour time point after ingesting taheebo extracts for 2 weeks. In conclusion, we demonstrated that administration of Tabebuia avellanedae inhibited ROS production from human neutrophils.

Keywords: Tabebuia avellanedae; Oxidative Stress; Neutrophil; Luminol-dependent Chemiluminescence; Inflammation; Mebiol Gel.

\section{*Corresponding Author:}

Katsuhiko Suzuki, M. D. \& Ph. D,

Professor of the Faculty of Sport Sciences, Waseda University, 2-579-15,

Mikajima, Tokorozawa, Saitama 359-1192, Japan.

Tel: +81-4-2947-6898

Fax: +81-4-2947-6898

E-mail: katsu.suzu@waseda.jp

Received: October 23, 2015

Accepted: November 18, 2015

Published: November 19, 2015

Citation: Suzuki K, et al., (2015) Ingestion of Tabebuia avellanedae (Taheebo) Inhibits Production of Reactive Oxygen Species from Human Peripheral Blood Neutrophils. Int J Food Sci Nutr Diet. S6:001, 1-4. doi: http://dx.doi.org/10.19070/2326-3350-SI06001

Copyright: Suzuki $\mathbf{K}^{\circ}$ 2015. This is an open-access article distributed under the terms of the Creative Commons Attribution License, which permits unrestricted use, distribution and reproduction in any medium, provided the original author and source are credited.

\section{Introduction}

Reactive oxygen species (ROS) play a dual role in biological systems that can be harmful or beneficial to living systems [1]. The beneficial effects of ROS include key roles of ROS in cellular responses in host defense against infectious agents and in a number of cellular signaling systems. In contrast, excessive ROS can be important mediators of damage to cell structures, including lipids, proteins and nucleic acids. Furthermore, these ROS-induced damages may lead to the effects of aging and a variety of diseases such as chronic inflammatory disease, cardiovascular disease and cancer $[2,3]$.

Neutrophils are a main source of ROS production, especially in inflammatory reactions. Neutrophils play an important role in host defense through phagocytosis against invading pathogens and are the major effectors of acute inflammatory reactions. In response to various agents, neutrophils release large quantities of superoxide anion $\left(\mathrm{O}_{2}^{-}\right)$in a phenomenon known as respiratory burst [1]. The inappropriate activation of respiratory burst is associated with tissue injury and impairs the ability of the immune system to remove invading microorganisms from the body. Thus, the balance between the beneficial and harmful effects of neutrophil functions need to be properly modulated.

Functional natural products, which suppress the production or promote the scavenging of ROS, have received much attention. One of such functional natural products is Tabebuia avellanedae, which is a broadleaf tree that grows wild in South America e.g., Brazil and the Amazonian watershed. The water extract of the inner bark of Tabebuia avellanedae was called "taheebo" and has been drunk as tea traditionally. Tabebuia avellanedae has also been used for various ethopharmacological treatments of bacterial infection, blood coagulation and cancer [4, 5]. Moreover, recent studies have shown that Tabebuia avellanedae has some anti-inflammation and anti-oxidation properties [6-8].

Although the anti-inflammatory effects of Tabebuia avellanedae have been reported in vitro and in animals, little is known about the effects of this natural product in humans. There is also no report that Tabebuia avellanedae inhibits neutrophil activation. To address these issues, the purpose of this study was to investigate 
the modulating effects of Tabebuia avellanedae extract on neutrophil function in humans by assessment of luminol-dependent chemiluminescence (LmCL). LmCL largely detects myeloperoxidase (MPO)-dependent formation of highly toxic ROS such as hypochlorous acid (HOCl) $[9,10]$.

\section{Materials and Methods}

\section{Subjects}

The inclusion criteria for this study were as follows: healthy adults; no use of dietary supplements; no severe organ function impairment and no chronic diseases. Prior to enrollment, all subjects signed a written informed consent. The protocol for this study was approved by the Ethical Committee at Waseda University.

\section{Study protocols}

The chosen test product, "TAHEEBO NAFDIN ${ }^{\circledR}$ soft capsule (Taheebo Japan, Co., Ltd., Osaka, Japan)", contains $125 \mathrm{mg}$ of Tabebuia avellanedae extract per capsule. One capsule contains the following: carbohydrates (127.5 mg), protein (115 mg), fat (195 $\mathrm{mg})$, sodium (0.41 mg), water (12.5 mg), Ca (0.71 mg), Fe (0.04 $\mathrm{mg}), \mathrm{K}(0.93 \mathrm{mg}), \mathrm{P}(0.20 \mathrm{mg})$, and $\mathrm{Mg}(0.28 \mathrm{mg})$. Results from tests for heavy metals (mercury, cadmium, lead and arsenic) conformed to strict Japanese food regulations. We did an open-label study in which 4 capsules of TAHEEBO NAFDIN ${ }^{\circledR}$ were ingested daily for 2 weeks. Peripheral blood was collected before administration of TAHEEBO NAFDIN@ ${ }^{\circledR}$ to establish an accurate baseline of ROS production by peripheral blood neutrophils. After administration of TAHEEBO NAFDIN ${ }^{\circledR}$, peripheral blood samples were obtained on the $1^{\text {st }}, 7^{\text {th }}$ and $14^{\text {th }}$ days.

\section{Synthesis of peptide-bound temperature-responsive poly- mer (G-TRP)}

Twenty-four grams of collagen peptide (SCP-5100; Nitta Gelatin Co., Osaka, Japan) were dissolved in $96 \mathrm{~g}$ of distilled water at $37^{\circ} \mathrm{C}$, followed by reaction with $3.26 \mathrm{~g}$ of $\mathrm{N}$-acryloylsuccinimide (Kokusan Kagaku Co., Ltd., Tokyo, Japan) for 4 days at $37^{\circ} \mathrm{C}$ to obtain polymerizable collagen peptide. N-Isopropylacrylamide (108.5 g; Kojin Co., Tokyo, Japan) and n-butyl methacrylate (4.26 g; Wako Pure Chemical Industries, Ltd., Osaka, Japan) were dissolved in $600 \mathrm{ml}$ of ethanol and then $123 \mathrm{~g}$ of the above aqueous solution of polymerizable collagen peptide was added. Under nitrogen atmosphere, $1 \mathrm{ml}$ of $\mathrm{N}, \mathrm{N}, \mathrm{N}, \mathrm{N}^{\prime}$ - tetramethylethylenediamine was added to the mixed solution (Wako Pure Chemical Industries, Ltd., Osaka, Japan) and $10 \mathrm{ml}$ of $10 \mathrm{wt} \%$ ammonium persulfate (Wako Pure Chemical Industries, Ltd., Osaka, Japan) aqueous solution were added and then reacted for 5 hours at $4^{\circ} \mathrm{C}$, maintaining the nitrogen atmosphere. After the reaction, $30 \mathrm{~L}$ of cold $\left(4^{\circ} \mathrm{C}\right)$ distilled water were added and the mixture concentrated to $3 \mathrm{~L}$ using an ultrafiltration membrane (molecular weight cut off of 100,000$)$ at $4^{\circ} \mathrm{C}$. This dilution and concentration process was repeated 5 times in order to remove impurities and low molecular species. Lyophilization and sterilization of the final concentrated solution gave $105 \mathrm{~g}$ of peptide-bound temperatureresponsive polymer $(\mathrm{G}-\mathrm{TRP})$.

\section{Preparation of scaffold-thermoreversible gelation polymer (S-TGP) gel}

Under a clean-air laminar hood workbench, $0.5 \mathrm{~g}$ of G-TRP and
$0.5 \mathrm{~g}$ of thermoreversible gelation polymer (Mebiol Gel; Mebiol Inc, Kanagawa, Japan) was dissolved in $16.7 \mathrm{ml}$ of Hank's Balanced Salt Solution (HBSS, calcium chloride, magnesium chloride) at $4^{\circ} \mathrm{C}$ overnight, yielding a viscous transparent scaffoldthermoreversible gelation polymer (S-TGP) gel of uniform liquid without any bubbles for use in the experiments [11]. Mebiol Gel is a pure synthesized biocompatible copolymer composed of thermoresponsive polymer blocks and hydrophilic polymer blocks, and is characterized by its temperature-dependent dynamic viscoelastic properties, which is used as a biocompatible scaffold for three-dimensional culture without any toxicity. S-TGP gel is a peptide-bound thermoreversible gel formed by mixing Mebiol Gel and G-TRP.

\section{Luminol-dependent chemiluminescence (LmCL) assay}

Peripheral blood samples were obtained from subjects using Na-heparin containing tubes (Terumo Venoject II, Terumo Co, Tokyo, Japan). An aqueous solution of S-TGP gel was solidified by raising its temperature. Accordingly, $50 \mu \mathrm{l}$ S-TGP gel was dispensed into microtubes $(2 \mathrm{ml})$, spread carefully at $4^{\circ} \mathrm{C}$, and set on block incubators at $37^{\circ} \mathrm{C}$. In addition, the blood samples were mixed with $2.5 \mathrm{mM}$ luminol (5-amino-2, 3-dihydro-1, 4-phthalazinedione; Sigma Aldrich, MO, USA) at a ratio of 1:1. The luminol-blood samples $(150 \mu \mathrm{l})$ were set on the S-TGP gel tubes at $37^{\circ} \mathrm{C}$. The production of ROS from neutrophils were detected as the values of LmCL using a luminometer (Gene Light 55; Microtec Co., Ltd., Funabashi, Japan), in a kinetic mode at 0, 0.5, 1.0 and 1.5 hours. Neutrophils migrate from the blood into the S-TGP gel in the tube at $37^{\circ} \mathrm{C}$, and LmCL can be detected through the transmissive gel. Therefore, there is no need to separate neutrophils from blood to determine ROS production, which reduces any delay in sample processing that is associated with conventional methods [9]. After LmCL was measured at 1.5 hours, luminolblood samples in the tubes were removed and the tubes with 50 $\mu$ l S-TGP in which neutrophils migrated were washed three times with PBS warmed at $37^{\circ} \mathrm{C}$. The tubes with gel were then cooled on ice, and $50 \mu \mathrm{l}$ reagent $\mathrm{A}$ and $50 \mu \mathrm{l}$ reagent $\mathrm{B}$ (ChemoMetec A/S, Allerød, Denmark) were added and mixed well, which effectively makes the cell membrane permeable to the DNA staining dye, and is effective in the dispersion of cell aggregates. The samples were aspirated into a NucleoCassett and the cell number was counted by the NucleoCounter (ChemoMetec A/S, Allerød, Denmark).

\section{Statistical analysis}

The Shapiro-Wilk test was used to check for normality of distribution. Statistical variations were analyzed with the Friedman repeated measures analysis of variance on ranks and with post-hoc multiple pairwise comparison for Friedman. Calculations were performed using IBM SPSS Statistics version 19.

\section{Results}

\section{Subjects' characteristics}

There were 14 subjects who enrolled for the study and their mean age was 43 years old, ranging from 30 to 57 . The study sample comprised 2 males and 12 females.

The LmCL values at each point 
Figure 1-7. Box and whisker plots demonstrating luminol-dependent chemiluminescence (LmCL) and cell counts in samples from volunteers over 2-week intake of taheebo: changes for 2 weeks of LmCL at 0.5 hours (1), 1.0 hours (2), 1.5 hours (3), peak value during measurement (4), sum of values at 1.5 hours (5), count of migratory cells into gel at 1.5 hours (6) and sum of values per a cell at 1.5 hours (7). P-values were calculated by post-hoc multiple pairwise comparison for Friedman. Boxplots indicate values between $25^{\text {th }}$ and $75^{\text {th }}$ percentiles of $\mathrm{LmCL}$ (central line, median). Vertical lines represent the $10^{\text {th }}$ and $90^{\text {th }}$ percentiles.
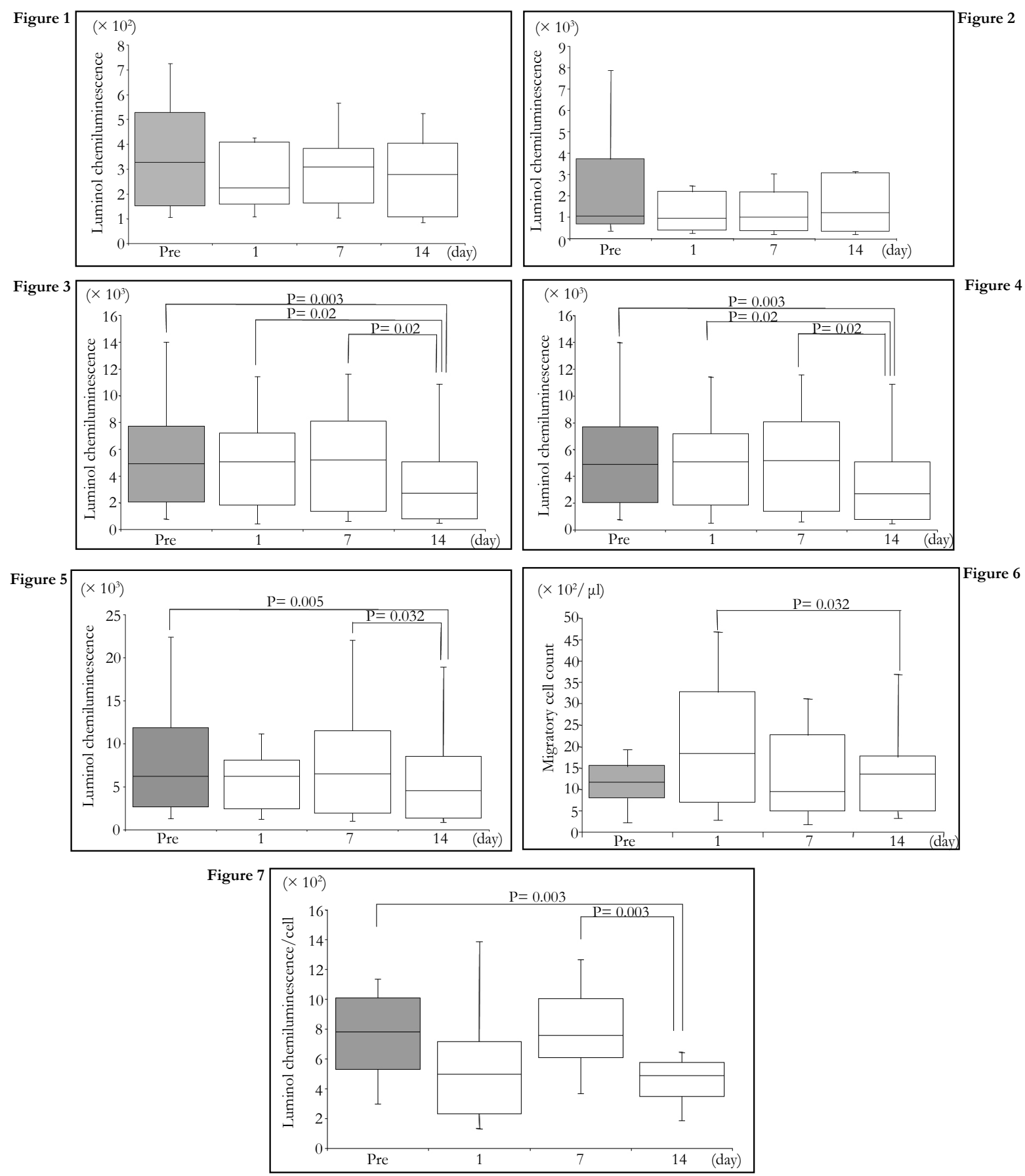

The LmCL values at 0.5 and 1.0 hour points were not significantly changed by 2 weeks of TAHEEBO NAFDIN ${ }^{\circledR}$ intake (Figures 1 and 2). The LmCL value at 1.5-hour point was markedly decreased by 2 -week intake of TAHEEBO NAFDIN ${ }^{\circledR}$ (Friedman: $\mathrm{P}=0.002)$ (Figure 3).

\section{Peak value in $\mathrm{LmCL}$}

The peak value in LmCL was assessed during measurement (0.5-1.5 hours) and was markedly decreased in the volunteers by 2-week intake of TAHEEBO NAFDIN ${ }^{\circledR}$ (Friedman: P $=0.004$ )
(Figure 4).

Sum of value in $\mathrm{LmCL}$

The total LmCL was determined from the sum of $\mathrm{LmCL}$ values at 0.5-1.5 hours. Total LmCL at 1.5 hours was significantly decreased by 2 -week intake of TAHEEBO NAFDIN ${ }^{\circledR}$ (Friedman: $\mathrm{P}=0.002$ ) (Figure 5).

\section{Number of migratory neutrophils}

The number of migrated cells was not significantly influenced 
by 2 -week administration of TAHEEBO NAFDIN ${ }^{\circledR}$ (Figure 6). When adjusted by migrated cell count, the LmCL values per cell basis were still inhibited by TAHEEBO NAFDIN ${ }^{\circledR}$ intake for 2 weeks (Friedman: $\mathrm{P}<0.001)$ (Figure 7).

\section{Discussion}

This study demonstrated that the LmCL values were significantly decreased in the volunteers after 2 -week intake of Tabebuia avellanedae extract. This effect of the extract remained significant even after adjusting for the number of migratory cells. These results suggest that Tabebuia avellanedae did not inhibit neutrophil migration, but the ROS produced by migrated cells was significantly suppressed.

The neutrophil activation may be regulated by Tabebuia avellanedae through the anti-inflammatory or the antioxidant effects. Limited reports are available on the anti-inflammatory effect of Tabebuia avellanedae. Byeon et al. demonstrated that Tabebuia avellanedae negatively modulated macrophage-mediated inflammatory responses by suppressing $\mathrm{PGE}_{2}$ production [6]. Awale et al. reported that Tabebuia avellanedae suppressed the activity of macrophage-like J774.1 cells [8]. Neutrophils play an important role like macrophages in inflammation. However, most studies did not focus on neutrophil functions controlled by Tabebuia avellanedae. We demonstrated that Tabebuia avellanedae inhibits the production of ROS in human neutrophils.

The major active compounds of Tabebuia avellanedae are furanonaphthoquinones, quinones (lapachol and $\beta$-lapachone), naphthoquinones, flavonoids, iridoids and phenolic glycosides. Iridoids and phenylethanoid glycoside was shown to inhibit the activities of nitric oxide (NO) production in LPS-activated macrophagelike J774.1 cells [8]. Furano naphthoquinones and $\beta$-lapachone may mediate immune modulating activities in vitro [12]. Although these compounds of Tabebuia avellanedae may influence neutrophil activation, additional research is needed to determine which compound controls the activation of neutrophils.

In this study, we demonstrated that Tabebuia avellanedae suppressed the activation of neutrophils in humans by LmCL assessment. Neutrophil respiratory burst activity can be measured in the presence of a light emitting reporter molecule by the production of NADPH oxidase-dependent oxidants such as $\mathrm{O}_{2}^{-}$, hydrogen peroxide $\left(\mathrm{H}_{2} \mathrm{O}_{2}\right)$ and MPO-dependent hypochlorous acid $(\mathrm{HOCl})$ production using luminol as the lumiphor [13]. We used Mebiol gel as thermoreversible gelation polymer, which is useful because this polymer liquefy at low temperature and turns to gel immediately upon warming before returning to a liquid state again when cooled. The greatest benefit of our method is its rapidity and simplicity of analysis using an analytical protocol, which does not require any cell separation procedure. In addition, our assay system, which uses the cellular response of neutrophils, to assess the state of neutrophil activation within the milieu of the immunologic profile may be applicable for screening antioxidant materials for estimating anti-inflammatory and/or antioxidant activity [14]. These insights potentially introduces novel anti-inflammatory therapeutic approaches to inflammation-related disease and disorder [15].
We demonstrated that Tabebuia avellanedae extract inhibited ROS production of neutrophils in humans. Future studies should focus on investigating the mechanisms and active substance of Tabebuia avellanedae in the context of neutrophil functional modulation.

\section{Acknowledgements}

We would like to thank Associate Professor Fabian Lim (Nanyang Technological University, Singapore) for editing the language and grammar of the manuscript, Ms Miki Tomari (Waseda University) and medical staffs of Maeda Hospital (Sayama City, Saitama, Japan) for assistance and coordination of clinical research. This work was partly supported by research funds endowed to Professor Katsuhiko Suzuki and visiting Associate Professor Satoshi Ohno from Taheebo Japan, Co., Ltd., Osaka, Japan. This publication was also made possible by a Grant-in-Aid for the Strategic Research Foundation at Private Universities from the Ministry of Education, Culture, Sports, Science and Technology of Japan. The funders had no role in study design, data collection and analysis, decision to publish, or preparation of the manuscript.

\section{References}

[1]. Oliveira BF, Nogueira-Machado JA, Chaves MM (2010) The role of oxidative stress in the aging process. Scientific World Journal 10:1121-1128.

[2]. Chen AF, Chen DD, Daiber A, Faraci FM, Li H, et al. (2012) Free radical biology of the cardiovascular system. Clin Sci (Lond) 123(2): 73-91.

[3]. Carnero A (2012) MAP17 and the double-edged sword of ROS. Biochim Biophys Acta 1826(1): 44-52.

[4]. Machado TB, Pinto AV, Pinto MC, Leal IC, Silva MG, et al. (2003) In vitro activity of Brazilian medicinal plants, naturally occurring naphthoquinones and their analogues, against methicillin-resistant Staphylococcus aureus. Int J Antimicrob Agents 21(3): 279-284.

[5]. Gómez Castellanos JR, Prieto JM, Heinrich M (2009) Red Lapacho (Tabebuia impetiginosa)--a global ethnopharmacological commodity? J Ethnopharmacol 121(1): 1-13.

[6]. Byeon SE, Chung JY, Lee YG, Kim BH, Kim KH, et al. (2008) In vitro and in vivo anti-inflammatory effects of taheebo, a water extract from the inner bark of Tabebuia avellanedae. J Ethnopharmacol 119(1): 145-152..

[7]. Böhler T, Nolting J, Gurragchaa P, Lupescu A, Neumayer HH, et al. (2008) Tabebuia avellanedae extracts inhibit IL-2-independent T-lymphocyte activation and proliferation. Transpl Immunol 18(4): 319-323.

[8]. Awale S, Kawakami T, Tezuka Y, Ueda JY, Tanaka K, et al. (2005) Nitric oxide (NO) production inhibitory constituents of Tabebuia avellanedae from Brazil. Chem Pharm Bull (Tokyo) 53(6): 710-713.

[9]. Hasegawa H, Suzuki K, Nakaji S, Sugawara K (1997) Analysis and assessment of the capacity of neutrophils to produce reactive oxygen species in a 96-well microplate format using lucigenin- and luminol-dependent chemiluminescence. J Immunol Methods 210(1): 1-10.

[10]. Suzuki Y, Ohno S, Okuyama R, Aruga A, Yamamoto M, et al. (2012) Determination of chronic inflammatory states in cancer patients using assay of reactive oxygen species production by neutrophils. Anticancer Res 32(2): 565-570.

[11]. Sudha B, Madhavan HN, Sitalakshmi G, Malathi J, Krishnakumar S, et al. (2006) Cultivation of human corneal limbal stem cells in Mebiol Gel - A thermo-reversible gelation polymer. Indian J Med Res 124(6): 655-664.

[12]. Kreher B, Lotter H, Cordell GA, Wagner H (1988) New furanonaphthoquinones and other constituents of Tabebuia avellanedae and their immunomodulating activities in vitro. Planta Med 54(6): 562-563.

[13]. Romaschin AD, Foster DM, Walker PM, Marshall JC (1998) Let the cells speak: Neutrophils as biologic markers of the inflammatory response. Sepsis 2: $119-125$.

[14]. Suzuki K, Ohno S, Suzuki Y, Ohno Y, Okuyama R, et al. (2012) Effect of green tea extract on reactive oxygen species produced by neutrophils from cancer patients. Anticancer Res 32(6): 2369-2375.

[15]. Sugama K, Suzuki K, Yoshitani K, Shiraishi K, Miura S, et al. (2015) Changes of thioredoxin, oxidative stress markers, inflammation and muscle/renal damage following intensive endurance exercise. Exerc Immunol Rev 21: $130-142$

\section{Conclusion}

